\title{
The Landscape of Micro-Inversions Provide Clues for Population Genetic Analysis of Humans
}

\author{
$\mathrm{Li} \mathrm{Qu}^{1,2} \cdot$ Luotong Wang ${ }^{1,3} \cdot$ Feifei He $\mathrm{He}^{1,3} \cdot$ Yilun $\mathrm{Han}^{1,3} \cdot$ Longshu Yang $^{1,3} \cdot$ May D. Wang $^{2} \cdot$ Huaiqiu Zhu ${ }^{1,2,3}$ (D)
}

Received: 25 March 2020 / Revised: 2 September 2020 / Accepted: 3 September 2020 / Published online: 14 September 2020

(c) The Author(s) 2020

\begin{abstract}
Background Variations in the human genome have been studied extensively. However, little is known about the role of microinversions (MIs), generally defined as small ( $<100 \mathrm{bp}$ ) inversions, in human evolution, diversity, and health. Depicting the pattern of MIs among diverse populations is critical for interpreting human evolutionary history and obtaining insight into genetic diseases.

Results In this paper, we explored the distribution of MIs in genomes from 26 human populations and 7 nonhuman primate genomes and analyzed the phylogenetic structure of the 26 human populations based on the MIs. We further investigated the functions of the MIs located within genes associated with human health. With hg 19 as the reference genome, we detected 6968 MIs among the 1937 human samples and 24,476 MIs among the 7 nonhuman primate genomes. The analyses of MIs in human genomes showed that the MIs were rarely located in exonic regions. Nonhuman primates and human populations shared only 82 inverted alleles, and Africans had the most inverted alleles in common with nonhuman primates, which was consistent with the "Out of Africa" hypothesis. The clustering of MIs among the human populations also coincided with human migration history and ancestral lineages.

Conclusions We propose that MIs are potential evolutionary markers for investigating population dynamics. Our results revealed the diversity of MIs in human populations and showed that they are essential to construct human population relationships and have a potential effect on human health.
\end{abstract}

Keywords Micro-inversions $\cdot$ Structural variations $\cdot$ Genome $\cdot$ High-throughput sequencing $\cdot$ Evolution

\section{Background}

As a kind of structural variation (SV) in genomes, inversions are defined as chromosome rearrangements in which a segment of a chromosome is reversed end to end [1]. Usually,

Electronic supplementary material The online version of this article (https://doi.org/10.1007/s12539-020-00392-6) contains supplementary material, which is available to authorized users.

Huaiqiu Zhu

hqzhu@pku.edu.cn

1 State Key Laboratory for Turbulence and Complex Systems and Department of Biomedical Engineering, College of Engineering, Peking University, Beijing 100871, China

2 Wallace H. Coulter Department of Biomedical Engineering, Georgia Tech and Emory University, Atlanta, GA 30332, USA

3 Center for Quantitative Biology, Peking University, Beijing 100871, China an inversion occurs when a single chromosome undergoes breakage and rearrangement within itself. Although it has long been known that inversions are associated with primate evolution [2], they were only recently found to play an important role in human evolution and diseases $[3,4]$. A number of studies have focused on inversions in the human genome [5, 6], and for decades, many detectable macroinversion polymorphisms in humans have been verified by experiments and proved to have been significant in human evolutionary history [7, 8]. For example, inversions located in 8 p23.1 have been reported to be associated with autoimmune and cardiovascular diseases [3]. In addition, inversions have been designated as evolutionary markers of human phenotypic diversity $[9,10]$. For example, a common $900 \mathrm{~kb}$ inversion polymorphism at $17 \mathrm{q} 21.31$ associated with Parkinson's disease suggested multiple distributions of inversions among ethnic populations [7]. Indeed, with the development of inversion detection methods, inversions are 
being increasingly recognized as one of the most important mechanisms underlying genetic diversity.

However, the studies mentioned above were mainly limited to the detection of large-scale inversions, usually $>100 \mathrm{~kb}$ in length. Recently, small-scale inversions (with lengths much shorter than $10 \mathrm{~kb}$ ) have been used in several studies for purposes, such as phylogenetic inference. These studies found an influence of small inversions on the formation of unusual flanking sequences in human and chimpanzee genomes [11] and developed tools to detect in-place inversions [12]. Nevertheless, the results of these studies were highly variable because of differences in the size used to define small inversions [13]. Moreover, current studies focus mainly on the differences in small inversions between human (Homo sapiens) and chimpanzee (Pan troglodytes) genomes [11], excluding the genomes of more distantly related primates, such as gorillas (Gorilla), orangutans (Pongo pygmaeus), gibbons (Hylobates sp.), baboons (Papio anubis), and rhesus macaques (Macaca mulatta).

Among all the inversions of small size, the microinversions (MIs) [13], a type of extremely small inversions (generally $<100 \mathrm{bp}$ ), which are found remarkably in human genomes, are uncertain with their function for the research community. However, statistical analysis suggested that MIs, usually similar to other rare genomic changes, may serve as phylogenetic markers because of their rare occurrence and low homoplasy [14]. Furthermore, many studies have examined the functions of inversions among multiple nonprimate species, such as yeast, sticklebacks, grasshoppers, Drosophila, ducks, chicken, and mice [15-21]. Regarding primates, which have larger genomes, comparative studies of the influence of chromosomal inversions occurring within chromosomes in the human and chimpanzee genomes can be traced back to the last century [2]. Nevertheless, such studies on human inversions are rare because of the limitations of detection techniques [22]. Great efforts have been focused on developing experimental techniques to discover large-scale inversions, including methods based on single-cell sequencing [23, 24], read assembly [25], and probe hybridization and amplification [26]. However, these experimental methods are usually expensive, time-consuming, and not aimed at detecting small inversions. With the advent of next-generation sequencing (NGS), the 1000 Genomes Project (1KGP) has provided a large amount of whole-genome sequencing data for individuals from a variety of populations across the world [27, 28]. However, the short reads generated by NGS in the $1 \mathrm{KGP}$ are generally very short $(<100 \mathrm{bp})$, making both the detection and analysis of MIs difficult. In contrast to well-studied large inversions, which cause more abnormal short reads and, thus, are discovered more frequently based on NGS short reads, MIs have not yet been studied because it is difficult to detect MIs shorter than the read length, most of which are identified as unmapped reads and, thus, discarded before further analysis. However, a large proportion of the sequenced short reads in the 1KGP are unmapped short reads that may contain SVs, many of which could be MIs. For example, in NA18525, a low-coverage sequencing sample in the 1KGP, 608,982,899 short reads are mapped reads, while $360,144,413$ (37.2\% of the total) are unmapped reads. The Micro-inversion Detection (MID) method developed in our previous study [13] uses these unmapped reads to detect MIs with good performance. The algorithm of MID was designed based on a dynamic programming path-finding approach, which can efficiently and reliably identify MIs from unmapped short NGS reads. This process subsequently facilitates the analysis of MIs across many populations based on high-throughput sequencing data. Therefore, an increased understanding of the MI landscape across various human populations will facilitate comparative analyses among individuals by taking individual differences into account.

Although efforts have been made to analyze small inversions that are still $>100 \mathrm{bp}$ in non-human organisms [11, 12], comprehensive studies of the effects of MIs, which are $<100$ bp in length, on human diversity, evolution, and diseases in a large number of human genomes in the $1 \mathrm{KGP}$ are lacking. In this study, we set out to detect MIs and further investigate the roles of MIs in the diversity and evolution of 26 human populations and 7 non-human primate populations. Overall, we explored the distribution of MIs in all 26 populations from the 1KGP [27] and detected 6968 MIs within all 1937 human samples and 24,476 MIs in 7 non-human primate genomes. Furthermore, we analyzed the extent of MI diversity and built phylogenetic trees by inverted allele counts at both the population and species scales. These results indicated that MIs rarely occur in or near protein-coding genes and that only a few are common in both primate and human populations. They also show that Africans share the most inverted alleles with non-human primates, which is consistent with the "Out of Africa" hypothesis [29]. More importantly, the phylogenetic analysis demonstrates that MIs are sensitive evolutionary markers for categorizing all populations. The categories coincide with human migration history and ancestral lineages. The analysis also revealed possible functions of some MIs located within disease-causing genes. Thus, MIs should be considered for studies of human evolution.

\section{Results}

\subsection{Overview and Distribution of MIs}

Our previous work [13] focused on the detection of MIs in the initial phases of the $1 \mathrm{KGP}$, which encompassed fewer individuals from only 19 populations and was largely limited to the statistical analysis of the MI distribution and 
polymorphisms, despite the few disease-related genes available for analysis. Herein, we constructed a more integrated map of MIs, including extra and new samples from the $1 \mathrm{KGP}$ phase III data. In addition, compared with our previous work, the current study presents a more comprehensive analysis that emphasizes the population diversity of MIs, quantifies the genetic impact of MIs, and explores the important roles that MIs play in human evolution. The flowchart of the entire analysis presented in this study is shown in Fig. 1.

Although the recurrence of specific MIs is rare, a series of MIs often converge to particular regions in the genome. To provide a more intuitive analysis of MIs, by extending the boundaries of overlapping MIs, we defined MI regions (MIRs) similar to the copy number variation (CNV) regions proposed by Yang et al.[30]. Thus, the MIRs refer to the united regions of overlapping MIs, with the requirement that an MIR has to be at most four base pairs longer than any one of its included MIs (shown in Fig. 2a). According to the definition of an MIR, each MI can be contained in only one MIR, and each MIR may contain at least one MI. Thus, the MIs in the same MIR have almost the same start and end positions on the chromosome. It should be noted that MIs merging in the same MIR in our article could not originate from the same individual because MIs with the same end limits detected in the same individual had been merged into one MI during the MID detection process [13]. Furthermore, the MIs merging in the same MIR were those in different individuals at the same region.

Since the MI could be considered a polymorphic variant that exists at a given position of the genome, one MI has two alleles. In this study, we refer to the allele found in the reference genome as the reference allele and the allele with the opposite inverted sequence against the reference genome as the inverted allele. We also refer to the inverted allele found in the common ancestor of the 7 non-human primates and the humans as the ancestral allele and the inverted allele, which appeared by mutation at some point over time, as the derived allele.

In this study, using the MID method [13], we identified 6968 MIs (see Table 1 for a list and statistics) among all unmapped reads in 1937 samples from the 26 human populations, which were merged into 2140 MIRs (the full list of sample names is provided in Table S1). Among the 6968 MIs, five occurred in more than 200 individual genomes. In addition, among the 2140 MIRs, 1169 (54.6\%) overlapped with gene regions, among which 1063 (90.9\%) overlapped with intronic and the remaining 106 overlapped with exonic regions (see Tables S2-S4 for the full list of

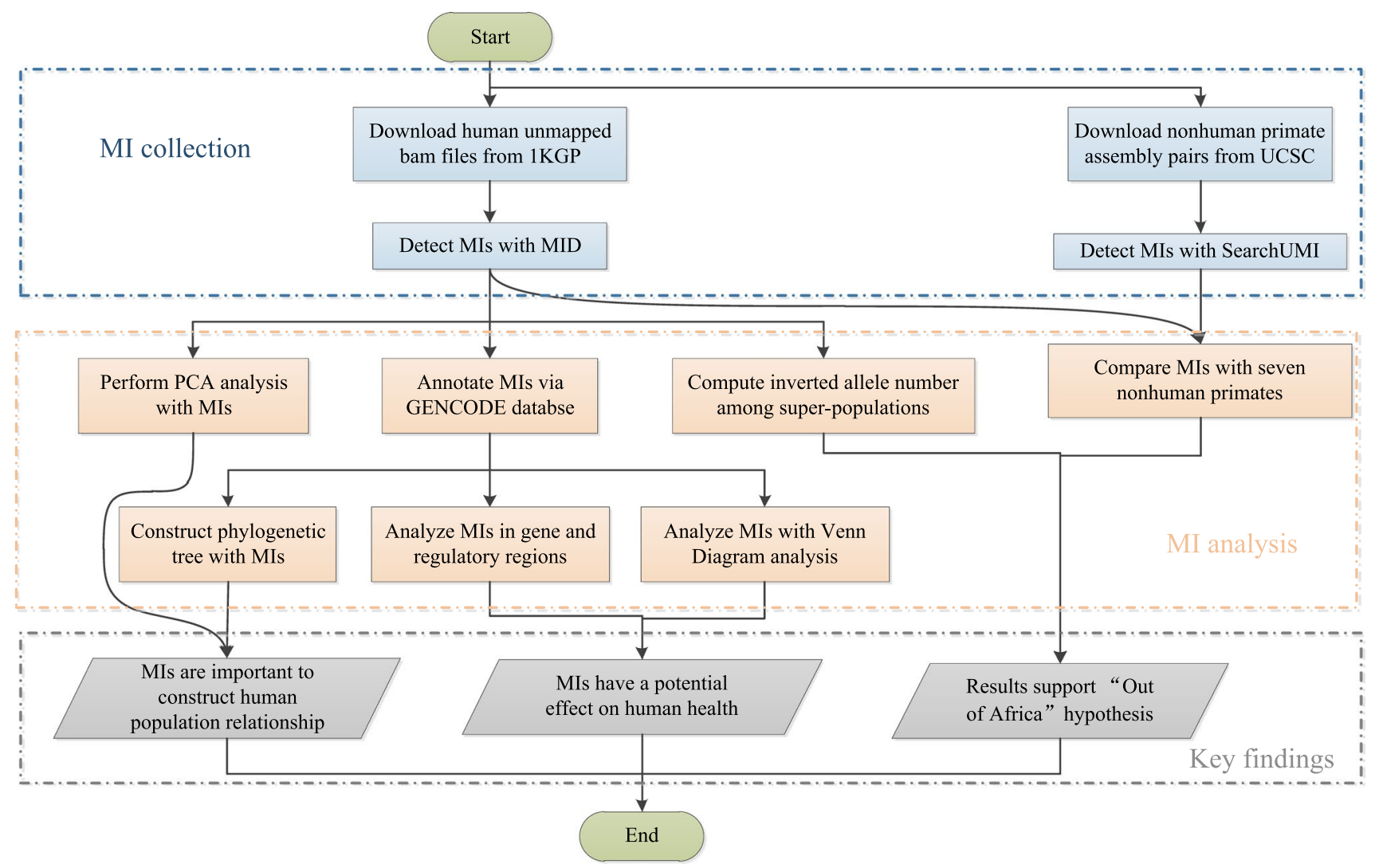

Fig. 1 A flowchart outlining the procedures of MI calling, MI analysis, and key findings 
Fig. 2 MIs and MIRs in human chromosomes. a Schematic showing how an MIR is a region where MIs overlap (black bars, MIRs; blue bars, MIs in one individual). b Scatter plot of MIR count against chromosome length. $\mathbf{c}$ Length distribution of 6968 MIs (13-45 bp) and 2,140 MIRs (14-45 bp). d Distribution of locations of MIs on chromosomes
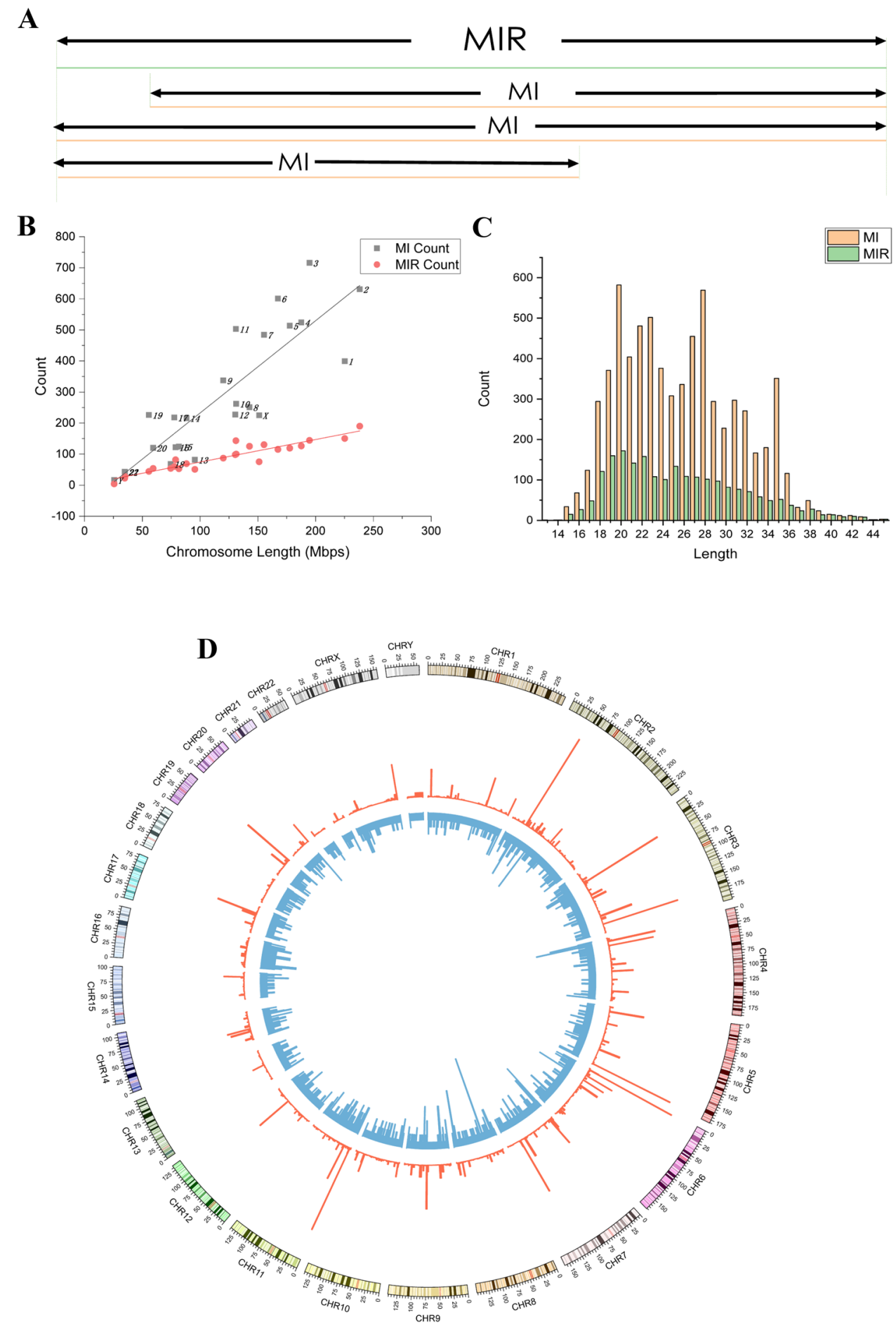

MIs, MIRs, and MIs in exonic regions, respectively). Of the 106 MIRs in exonic regions, 30 (28.3\%) overlapped with coding sequence (CDS) regions, 39 (36.8\%) with untranslated regions (UTRs), and 37 (34.9\%) with other functional regions, including miRNA and $\mathrm{mt}$ _rRNA regions. Of the 971 MIRs in intergenic regions, 47 MIRs (4.8\%) overlapped with candidate promoter regions.
Information regarding the $30 \mathrm{MIs}$ overlapping with coding sequence (CDS) regions is provided in Table S5. As shown in Table S5, most of the 30 MIs were located within CNV loss or gain regions. This result indicates that the MIs may affect gene function combined with other kinds of SVs. Additionally, 10 of the 30 MIs include SNPs according to the annotation of the dbSNP [31] or the Exome Aggregation Consortium (ExAC) [32] database. The bases marked 
Table 1 Overview of MIs detected in 1937 samples

\begin{tabular}{|c|c|c|c|c|c|c|c|}
\hline Pop & Population description & Sam-num & MIR-num & MI-num & Mul-sup & $\begin{array}{l}\text { MI-num/ } \\
\text { Sam-num }\end{array}$ & Mul-sup/MIR-num \\
\hline $\mathrm{CHB}$ & Han Chinese in Beijing, China & 86 & 119 & 202 & 30 & 2.35 & $25.21 \%$ \\
\hline JPT & Japanese in Tokyo, Japan & 104 & 209 & 416 & 61 & 4.00 & $29.19 \%$ \\
\hline CHS & Southern Han Chinese & 110 & 116 & 183 & 27 & 1.66 & $23.28 \%$ \\
\hline CDX & Chinese Dai in Xishuangbanna, China & 99 & 59 & 99 & 16 & 1.00 & $27.12 \%$ \\
\hline KHV & Kinh in Ho Chi Minh City, Vietnam & 101 & 176 & 249 & 32 & 2.47 & $18.18 \%$ \\
\hline$E A S$ & East Asia & 500 & 483 & 1149 & 110 & 2.30 & $16.2 \%$ \\
\hline CEU & $\begin{array}{l}\text { Utah Residents (CEPH) with Northern and } \\
\text { Western Ancestry }\end{array}$ & 100 & 300 & 387 & 37 & 3.87 & $12.33 \%$ \\
\hline TSI & Toscani in Italia & 98 & 137 & 195 & 23 & 1.99 & $16.79 \%$ \\
\hline FIN & Finnish in Finland & 99 & 237 & 283 & 18 & 2.86 & $7.59 \%$ \\
\hline GBR & British in England and Scotland & 92 & 185 & 251 & 31 & 2.73 & $16.76 \%$ \\
\hline IBS & Iberian Population in Spain & 108 & 199 & 271 & 31 & 2.51 & $15.58 \%$ \\
\hline EUR & Europe & 497 & 877 & 1387 & 92 & 2.79 & $10.49 \%$ \\
\hline YRI & Yoruba in Ibadan, Nigeria & 68 & 156 & 247 & 33 & 3.63 & $21.15 \%$ \\
\hline LWK & Luhya in Webuye, Kenya & 105 & 161 & 278 & 44 & 2.65 & $27.33 \%$ \\
\hline GWD & Gambians in Western Divisions in the Gambia & 113 & 177 & 384 & 65 & 3.40 & $36.72 \%$ \\
\hline MSL & Mende in Sierra Leone & 85 & 168 & 316 & 29 & 3.72 & $17.26 \%$ \\
\hline ESN & Esan in Nigeria & 99 & 167 & 347 & 51 & 3.51 & $30.54 \%$ \\
\hline ASW & Americans of African Ancestry in the SW USA & 66 & 149 & 293 & 50 & 4.44 & $33.56 \%$ \\
\hline $\mathrm{ACB}$ & African Caribbeans in Barbados & 96 & 152 & 281 & 51 & 2.93 & $33.55 \%$ \\
\hline$A F R$ & Africa & 632 & 634 & 2146 & 192 & 3.40 & $30.28 \%$ \\
\hline MXL & Mexican Ancestry from Los Angeles, USA & 67 & 93 & 176 & 26 & 2.63 & $27.96 \%$ \\
\hline PUR & Puerto Ricans from Puerto Rico & 57 & 107 & 220 & 46 & 3.86 & $42.99 \%$ \\
\hline CLM & Colombians from Medellin, Colombia & 94 & 163 & 267 & 31 & 2.84 & $19.02 \%$ \\
\hline PEL & Peruvians from Lima, Peru & 86 & 125 & 187 & 22 & 2.17 & $17.60 \%$ \\
\hline$A M R$ & America & 304 & 347 & 850 & 82 & 2.80 & $23.63 \%$ \\
\hline GIH & Gujarati Indians from Houston, Texas & 106 & 108 & 193 & 29 & 1.82 & $26.85 \%$ \\
\hline PJL & Punjabi from Lahore, Pakistan & 96 & 141 & 351 & 54 & 3.66 & $38.30 \%$ \\
\hline BEB & Bengali from Bangladesh & 86 & 131 & 270 & 36 & 3.14 & $27.48 \%$ \\
\hline STU & Sri Lankan Tamil from the UK & 103 & 139 & 334 & 51 & 3.24 & $36.69 \%$ \\
\hline ITU & Indian Telugu from the UK & 103 & 119 & 288 & 53 & 2.80 & $44.54 \%$ \\
\hline$S A S$ & South Asia & 494 & 377 & 1436 & 112 & 2.91 & $29.71 \%$ \\
\hline Total & - & 2427 & 2140 & 6968 & 248 & 2.87 & $11.59 \%$ \\
\hline
\end{tabular}

Pop population name; Sam-num the number of samples for the population; MIR-num the number of MIRs in each population; MI-num the number of inverted alleles in each population; Mul-sup the MIRs supported by at least two MIs; MI-num/Sam-num the ratio of the number of inverted alleles to the number of samples, which indicates the average inverted alleles per individual; Mul-sup/MIR-num the ratio of MIRs with multiple MIs to the total number of MIRs

in red color in the column "Reference allele sequence" and "Inverted allele sequence" are the SNP polymorphism positions contained in the MI sequences. It should be noted that the inverted allele of chr11: 126,146,988-126,147,016 included the SNP at position 126,147,003 (A > G) named rs7116126 by the dbSNP database, which was annotated by the ClinVar [33] database as a conflicting interpretations of pathogenicity. Thus, the pathogenicity of this SNP allele is not clear, and various studies have reported conflicting interpretations of this SNP. To further analyze this MI polymorphism, we listed the details of this MI annotated by multiple databases from the UCSC genome browser (https ://genome.ucsc.edu/) [34] in Fig. S4, showing that the MI region is conserved in a few vertebrates, such as the human, rhesus macaque, mouse, dog, elephant, and chicken, which indicates that this region may not change easily. Further evidence is needed to determine the specific function of this MI linked with a few SVs and SNP rs7116126.

A scatter plot of the inverted allele number and MIR count against chromosome length is shown in Fig. 2b. Generally, the numbers of inverted alleles and MIRs are positively correlated with the lengths of chromosomes (Fig. 2b), which is 
reasonable, because longer chromosomes have more DNA bases and, thus, a greater chance of DNA replication errors. Additionally, the correlations between MI and MIR counts and length were analyzed using Pearson's correlation analysis. Specifically, a strong correlation was detected between the MIR count and chromosome length, where the Pearson's correlation coefficient was $r=0.935(P<0.0001)$; the corresponding result for MI was $0.861(P<0.0001)$. A scatter plot of MI and MIR counts against gene density is shown in Fig. S1. Although we found no strong linear correlation between the number of inverted alleles and gene density, we found a phenomenon, in which most scatter above the line of best fit in Fig. 2b was attributable to chromosomes with high gene density. For example, we discovered that chromosomes 19,17, 22, and 11, which were responsible for the scatter above the line of best fit, have high gene density, as shown in Fig. S1. This suggests that gene density may also affect the inverted alleles of a chromosome, while this relationship is not strictly linear. The MI and MIR event rate distribution across chromosomes is shown in Fig. S2. As shown in Fig. 2c, the lengths of the MIs varied from 15 to $45 \mathrm{bp}$ but were concentrated within 18 to $30 \mathrm{bp}$. The specific positions of MIs among 24 chromosomes are shown in Fig. 2d. Notably, when comparing the MIs in our study with the inversion-calling results from the phase III analysis of the 1KGP, we found that the shortest inversion detected by the 1KGP was $257 \mathrm{bp}$, which was much longer than $100 \mathrm{bp}$. We also noted that in other studies on inversions, no MIs in the range from 10 to $100 \mathrm{bp}$ were discussed. This indicates a discrepancy in length between the MIs in this study and traditional inversions in previous studies. Thus, our analysis of MIs ( $<100 \mathrm{bp}$ ), overlooked by all the studies including $1 \mathrm{KGP}$ analysis, sheds light on the limited understanding of human SVs.

\subsection{Inverted Allele Number Per Individual Among Populations}

Single-nucleotide polymorphisms (SNPs) and SVs in the $1 \mathrm{KGP}$ have been reported to display variant polymorphism and reveal genetic diversity among 26 populations [27]. However, MIs, as a kind of SVs, have never been studied to capture the genetic diversity among 26 populations. Thus, we report an analysis of MI diversity in this subsection.

We calculated an average of 2.87 inverted alleles per individual for all individuals. A bar plot showing the average counts of inverted alleles per individual for five superpopulations (African, American, South Asian, East Asian, and European) is presented in Fig. S3, which shows that the African super-population had the most inverted alleles per individual at 3.4, while the East Asian population had the fewest at 2.3. As we focused more on the effect of inverted alleles but not reference alleles on evolution, we also displayed the average count of inverted alleles of the five super-populations without including the 490 samples with no MIs detected in Fig. 3a. This analysis showed that when considering only individuals with the inverted alleles, the five super-populations ranked in descending order according to the average count of inverted alleles per individual were as follows: African $>$ American $>$ European $>$ South Asian $>$ East Asian. Notably, the African super-population had the most inverted alleles per individual at 3.96, while the East Asian population had the fewest at 3.03. The results displayed in both Fig. S3 and Fig. 3a are consistent with those of recent studies employing human SNP analysis [27, 35], in which the African super-population has the largest SNP number and the East Asian super-population has the smallest SNP number. In addition, a phylogenetic tree was constructed according to the MIs in gene regions for the five super-populations (see Fig. 3b). The result implies that the African super-population is distinct from the other 4 superpopulations. The descending order of the super-populations based on average inverted allele number and the clustering result also fit the Out of Africa hypothesis [29], which posits that humans originated in Africa and then modern Africans migrated to other continents. We will discuss this point further in the Discussion and conclusions section. Regarding the 26 populations, the average inverted allele count per individual populations ranged from 1.00 to 4.44 (see Table 1). Furthermore, the population of Americans of African Ancestry in the SW USA (ASW) had the most inverted alleles at 4.44, and the Chinese Dai in Xishuangbanna, China (CDX) had the least at 1.00. The geographic locations of the 26 populations are shown in Fig. 3c.

\subsection{Population Structure Based on MI Statistics}

To examine the genetic structure of MIs hierarchically among populations, we constructed a genetic distancebased phylogenetic tree using all the MIs detected from the 26 populations (Fig. 4a), which yielded results that generally provided evidence of genetic clustering: African and American populations were clustered on one branch, European and South Asian populations were clustered on a second branch, and East Asian populations were clustered on a third branch. Since the gene regions are generally considered as conserved domains, the MIs therein may reflect the human evolution and population relationship better than in intergenic regions. Therefore, we also constructed a phylogenetic tree with only MIs in gene regions (Fig. 4b). Similar to the analysis of all MIs presented in Fig. 4a, the phylogenetic analysis with MIs in gene regions presented in Fig. 4b clustered the populations on four branches. The topology of the phylogenetic tree not only met our expectation, but also reflected a regional pattern among the 26 populations, suggesting that population 
A

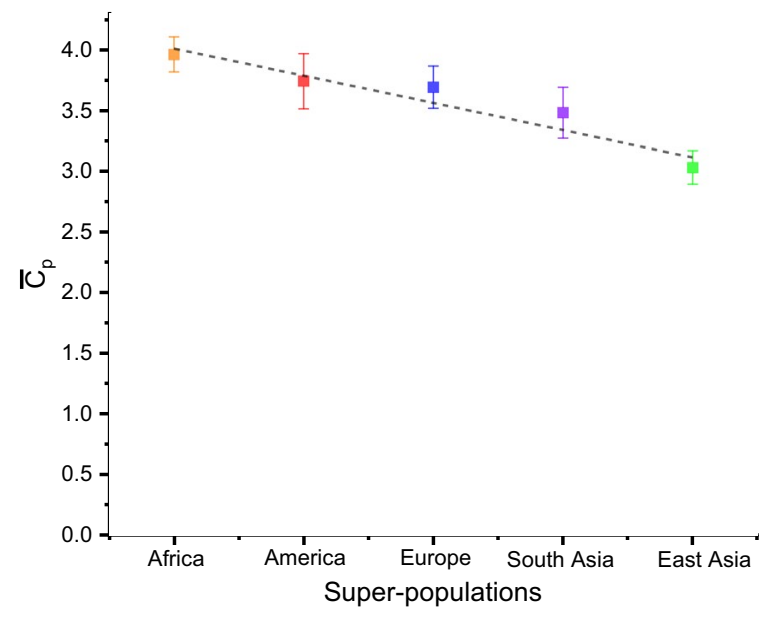

B

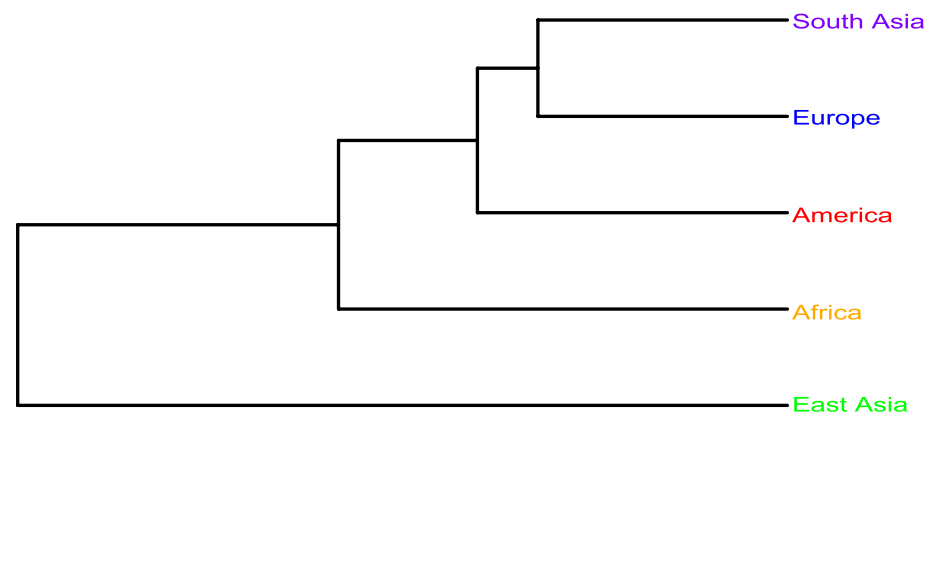

C

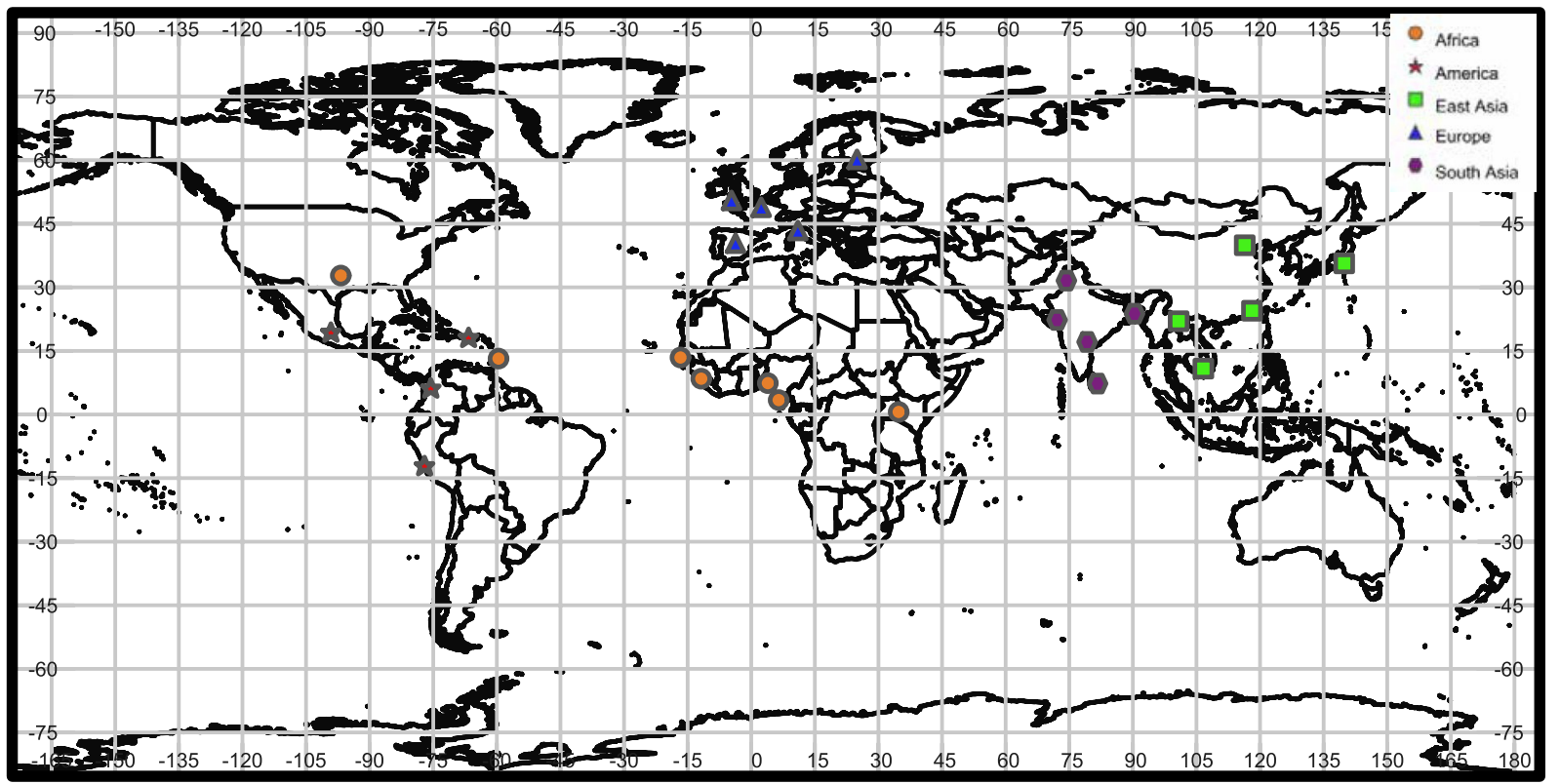

Fig. 3 Average counts of inverted alleles per individual. $\overline{C_{p}}$ is the average count of inverted alleles per individual. Different colors represent the five super-populations: orange, African; red, American; blue, European; purple, South Asian; and green, East Asian. a Bar plot of the average count of inverted alleles per individual among the five super-populations. The data are presented as the mean \pm standard error (SE). The average count ranged from 3.03 to 3.96. b Phylogenetic tree based on the average pairwise differences between superpopulations according to the MIs in gene regions. c Geographic locations of the 26 populations groups that live geographically closer to one another have shorter MI-based genetic distances. An exception was the Finnish in Finland (FIN) population (clustered with the East Asian populations in Fig. 4a and forming a single cluster in Fig. 4b), which deviated from the European branch. This deviation of FIN is understandable because Finland is genetically distant from the rest of the European populations [36]. In addition, Finnish are treated as a separate population rather than a European population in some studies [36]. The phylogenetic tree revealed that MIs with functions similar to those of SNPs are key to tracing the evolution of human population genetic structure. The matrices for constructing the phylogenetic trees presented in Fig. 4a, b are shown in Tables S6 and S7, respectively.

To further reveal undiscovered MI-based relationships among the 26 populations, we performed principal component analysis (PCA) of the 26 populations with all 6968 MIs. As shown in Fig. 5, the PCA of MI patterns divided the 26 populations into four groups according to the top two main components. This result indicated that populations in the same super-population were closer to each other than populations in different super-populations. We found that 
Fig. 4 Phylogenetic trees of 26 populations constructed by neighbor joining. a Tree for the 26 populations based on average pairwise population distances calculated with all 6968 MIs. b Tree based on average pairwise population distances calculated with the 2135 MIs in gene regions

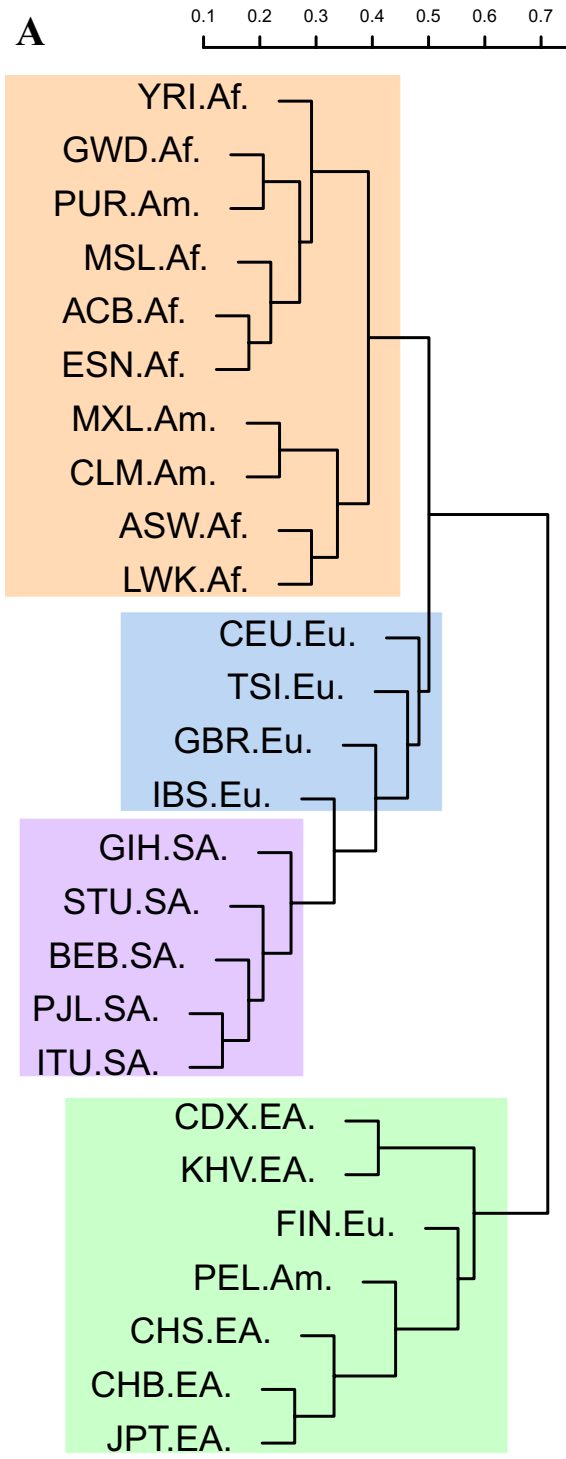

B

$\begin{array}{llllllll}0.1 & 0.2 & 0.3 & 0.4 & 0.5 & 0.6 & 0.7 & 0.8\end{array}$

FIN.Eu.

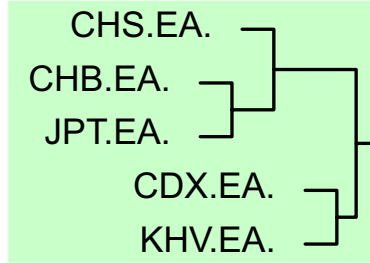

TSI.Eu.

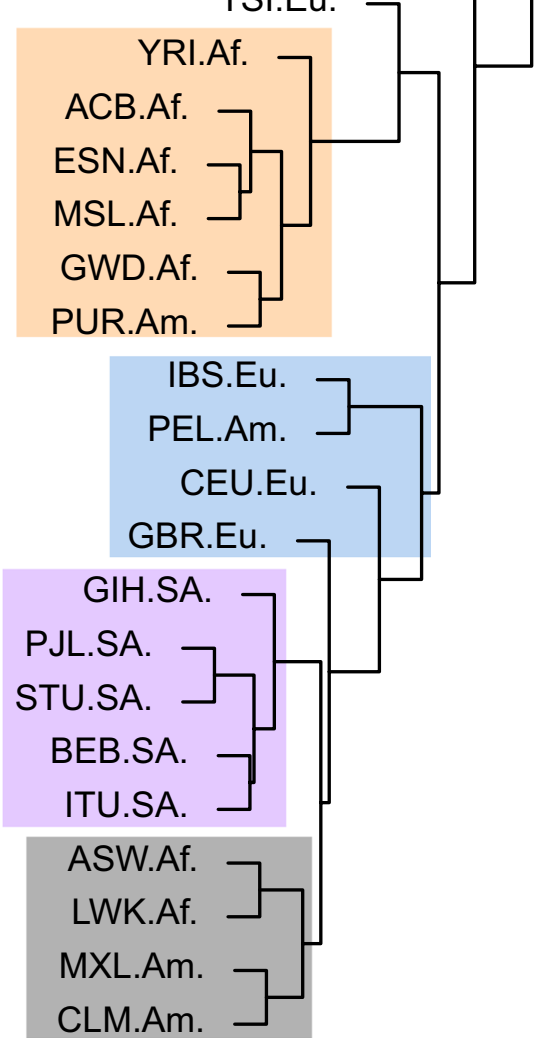

African, European, South Asian, and East Asian populations were clearly recognizable, which represented evolution via genetic drift or other factors. In contrast to those in the other four super-populations, the populations in the American super-population were dispersed and did not form a distinct cluster. Our results are highly consistent with the widespread pattern observed with PCA based on SVs [27].

\subsection{Analysis of MIR Sharing Among the Five Super-Populations}

Since the distinct variation pattern of each population may implicitly affect phenotypic divergence among the populations, it is important to explore the common and distinct MIRs among the five super-populations to see if there are differences in MIs among the super-populations. Therefore, to further investigate the diversity and relationships of MIs among the five super-populations, we conducted MIR sharing analysis with a Venn diagram by counting all the MIRs (Fig. 6a). We found that 76 MIRs were shared by all five super-populations. Furthermore, we used only the MIRs located in gene regions to construct the Venn diagram presented in Fig. 6b. A total of 34 MIRs within gene regions annotated by the GENCODE database were shared by all five super-populations (Fig. 6b). In addition, no MIRs in gene regions were shared by the American, European, South Asian, and East Asian super-populations, except for the 34 MIRs that were shared by all five super-populations. However, any four of the super-populations, as long as Africa was included, shared at least one MIR in addition to the 34. This result suggests that the American, European, South Asian, and East Asian super-populations are more closely related to the African super-population than to each other. 
Fig. 5 PCA of 26 populations. The first and second principal components are plotted. Different colors represent the five super-populations: orange, African; red, American; blue, European; purple, South Asian; and green, East Asian

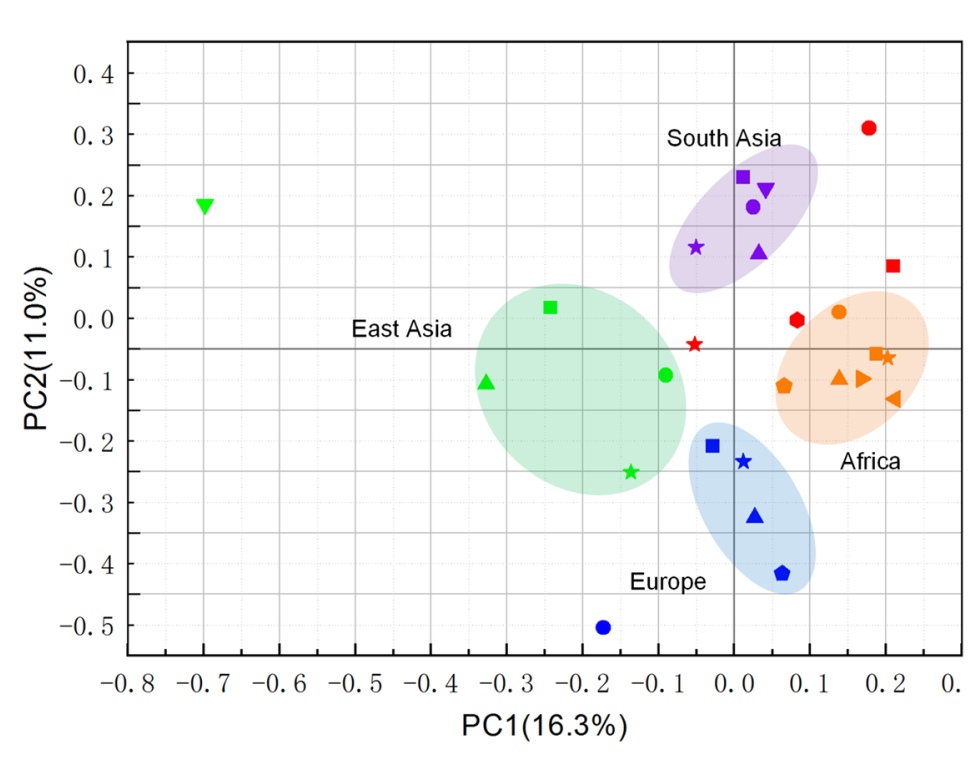

- CHB.ea

JPT.ea

$\triangle \mathrm{CHS}$.ea

$\checkmark$ CDX.ea

* KHV.ea

- CEU.eu

- TSI.eu

- FIN.eu

- GBR.eu

$\star$ IBS.eu

- ACB.af

- ASW.af

- ESN.af

A MSL.af

GWD.af

LWK.af

4 YRI.af

- MXL.am

- PUR.am

- CLM.am

* PEL.am

• GIH.sa

- PJl.sa

- BEB.sa

- STU.sa

$\checkmark$ ITU.sa

East Asia

Europe

Africa
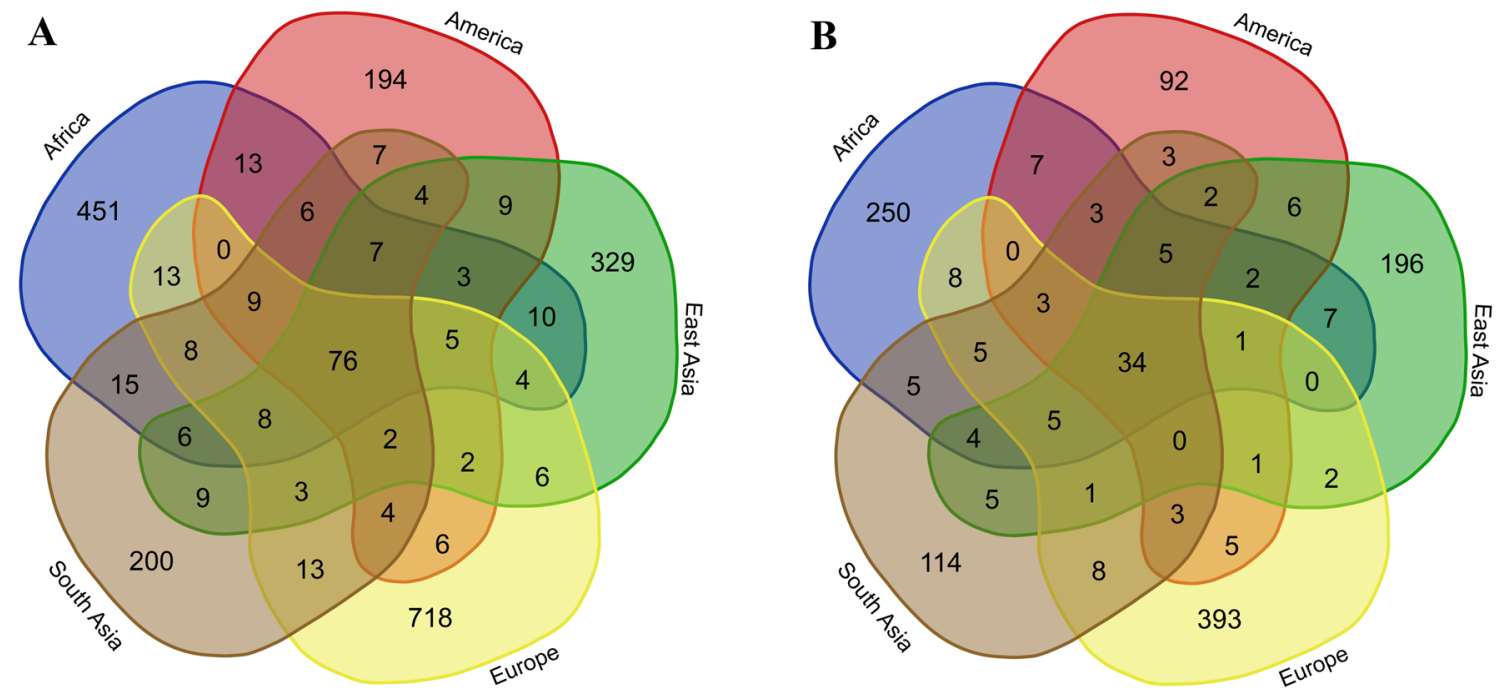

Fig. 6 Venn diagrams of MIR sharing among super-populations. a Diagram of all MIRs shared among the five super-populations. b Diagram of only MIRs in gene regions according to the GENCODE database shared among the five super-populations

In the current study, we defined an "MIR hit" as the number of MI alleles included within an MIR. Consequently, most MIRs in gene regions were unique in each superpopulation (i.e., singleton MIRs), especially the European population. There were a total of 1008 singleton MIRs and 36 non-singleton MIRs. The full list of MIR hit counts in gene regions among the super-populations is provided in Table S8. According to the results listed in Table S9, the most recurrent non-singleton MIR in gene regions had eight hits in the African super-population. The second most recurrent MIR (hits=7) was also detected in the African super-population.

\subsection{Potential Effects of Mls on Human Health}

Although the MIs in normal individuals have not immediately impacted or directly caused diseases, MIs occurring in gene regions rather than intergenic regions are more likely to affect gene function and, thus, potentially result in phenotypic diversity in disease susceptibility among various populations in the future. To investigate the potential association of MIs with gene function and human diseases among various populations, we conducted an analysis of the genes that were frequently affected by MIs. We mainly focused on the eight genes that overlapped with MIRs (Table S9) with $>3$ hits as listed in Table S8. Then, we obtained gene functions 
through GeneCard as well as related reference studies; the functions are listed in Table S9. As shown in Table S9, MIs cause substitutions at the same position in gene regions of the hg 19 human reference genome. Although it is not clear how these MIs in intronic or exonic (UTR) regions affect the functions of genes, it has been reported that both UTRs and exonic regions play an important role in translation and transcription [37, 38]. In addition, most of the genes listed in Table $\mathrm{S} 9$ have been reported to be related to health. Among these genes, ANKRD36 overlapped with MIs only in African populations. This gene is associated with genes that control the capacity to tolerate alcohol [39]. Besides this MI, the MI located in MIR between chr10:61,808,458-61,808,486 has caused an inverted sequence in one intron of Gene $A N K 3$, potentially affecting an element region, by which multiple sites are defined by ENCyclopedia of DNA Elements (ENCODE) [40] as being able to affect gene expression. According to ENCODE definition, this MI is included in a CTCF transcription factor (TF) binding site region between chr10:61,807,778-61,808,678, derived from a large collection of ChIP-seq experiments performed by the ENCODE project [40], which could potentially affect the gene expression of $A N K 3$. It has been reported previously that disease-related polymorphisms influence factor-binding site sequences in bipolar disease [41]. Besides, SNPs correlated with disease susceptibility in bipolar disease have been recorded to alter the regulation of ANK3 gene expression [42]. Considering the points above, the inverted allele between chr10:61,808,458-61,808,486, which causes 22 base substitutions in the intron region, may have some implications in terms of $A N K 3$ gene expression and function. In addition, $A N K 3$, which overlapped with MIs in only East Asians, is reportedly a risk factor for schizophrenia, a chronic and severe mental disorder, in Han Chinese [43], which may explain the existence of MIs in ANK3 only in East Asians.

\subsection{Comparison with 7 Non-Human Primates}

Since we detected an important role of MIs in humans, it is worthwhile to explore the impact of MIs on the association of humans with non-human primates. In contrast to the individual genomes of the human populations from the 1KGP, herein, we used the alignments of human and 7 closely related non-human primate (chimpanzee, gorilla, orangutan, gibbon, baboon, rhesus macaque, and squirrel monkey) genomes to analyze MIs due to the lack of individual nonhuman primate genomes. Subsequently, we detected the MIs in the 7 non-human primates by applying the searchUMI tool [44] (see Materials and methods section for detection details) and identified a total of 24,476 MIs with the hg 19 human assembly as a reference (Table S10). Notably, since MIRs were defined on the basis of the hg19 human genome assembly rather than the genome of the common ancestor of humans and the non-human primates, we adopted the MIs but not the MIRs for the non-human primates in this analysis.

Furthermore, we compared the MIs in humans with those in the 7 closely related non-human primates (chimpanzee, gorilla, orangutan, gibbon, baboon, rhesus macaque, and squirrel monkey) to investigate the inheritance and evolution of MI. By comparing inverted alleles in the genomes of non-human primates against the hg19 human reference genome and inverted alleles detected in the genomes of the 1KGP super-populations, we discovered a larger number of inverted alleles in African populations than in other populations that overlapped with those of the 7 non-human primates.

As shown in Table 2, the number of inverted alleles shared by non-human primates and the five super-populations in descending order were as follows: 58 shared with the African super-population, 49 with the East Asian superpopulation, 49 with the South Asian super-population, and 35 with the European super-population. The highest ratio of inverted allele overlap observed between the non-human primates and Africans might be because Africans inherited

Table 2 Counts of common inverted alleles among 7 non-human primates and five human super-populations

\begin{tabular}{|c|c|c|c|c|c|c|c|}
\hline Name & $\begin{array}{l}\text { All inverted } \\
\text { allele count }\end{array}$ & East Asian & South Asian & European & African & American & $1 \mathrm{KGP}$ \\
\hline Chimpanzee (panTro4) & 2284 & 12 & 11 & 7 & 14 & 11 & 20 \\
\hline Gorilla (gorGor3) & 2010 & 10 & 11 & 9 & 14 & 10 & 18 \\
\hline Orangutan (ponAbe2) & 3473 & 9 & 9 & 7 & 10 & 9 & 14 \\
\hline Gibbon (nomLeu1) & 3713 & 6 & 7 & 5 & 7 & 3 & 13 \\
\hline Baboon (PapHam1) & 4547 & 5 & 5 & 3 & 6 & 5 & 8 \\
\hline Rhesus macaque (rheMac3) & 4271 & 5 & 4 & 2 & 5 & 4 & 7 \\
\hline Squirrel monkey (SaiBol1) & 4214 & 2 & 2 & 2 & 2 & 2 & 2 \\
\hline Total & 24,476 & 49 & 49 & 35 & 58 & 44 & 82 \\
\hline
\end{tabular}


these shared inverted alleles from the common ancestor of non-human primates and humans which could be the ancestry alleles. Specifically, we speculate that after the Out of Africa geographic movement of modern humans, the African ancestral population spread the distribution of most ancestry alleles, and some of these ancestry alleles now survive in contemporary populations, resulting in the smaller inverted allele numbers in the other 4 super-populations. However, further sophisticated fossil and genetic data including the allele frequency based on more samples are necessary to support this conclusion.

\section{Discussion}

In this study, we performed a comprehensive analysis of MIs in individual genomes from the $1 \mathrm{KGP}$ and 7 non-human primate genomes, built an MI landscape for the human populations, and explored the effect of MIs on human diversity, evolution, and health. Applying the software MID [13] for 26 human populations and searchUMI [44] for alignments between the human and 7 non-human primate genomes, we analyzed a total of 6968 MIs detected in 1937 individuals of 26 populations from the $1 \mathrm{KGP}$ and 82 inverted alleles common among the 7 non-human primate genomes and the 1937 individuals. The MIs from the five super-populations (African, American, European, South Asian, and East Asian) revealed the population structure at multiple levels and may affect individuals in several ways. First, the widespread MI polymorphisms in human genomes greatly contribute to human diversity, which could be a result of geographical distribution and human migration. Second, large-scale MIs are good supplements to current evolutionary markers for reconstructing human evolutionary history. Third, the MIs in particular super-populations, especially the MIs overlapping with gene regions, are informative for understanding the association between genetic variations and health.

\subsection{Average Number of Mls in the Five Super-Populations}

We arranged the five super-populations in descending order based on the average number of inverted alleles per individual as follows: African $>$ American $>$ European $>$ South Asian $>$ East Asian (Fig. 3a). The average numbers of inverted alleles per individual among these five super-populations supported the Out of Africa hypothesis, which posits that humans originated in Africa and that recent Africans migrated to other continents. Other studies have speculated that modern Africans first migrated to Eurasia, which is the continent consisting of Europe and Asia [45]. In addition, the genetic distance between Africans and Europeans was reported to be smaller than that between Africans and
Asians, which implies a closer relationship between Africans and Europeans [45]. In addition, as reported by Macaulay et al.[46], the initial branch of the Asians from the southern dissemination followed the Nile from the east of Africa, went towards the north, and entered Asia. Once through the Sinai, the group of humans split, with some shifting into Europe and the others heading into Asia. This assumption is based on the comparatively late date of the landing of modern humans in Europe. Furthermore, the clustering result for the five super-populations based on MIs (Fig. 3b), which showed a tendency for European and Asian populations to cluster together and originate from Africans, also supported this hypothesis. In addition, East Asians were observed to have the greatest distance from Africans in the clustering results, which indicated that Asia was the last continent to which Africans arrived. It has been previously reported that during the migration to East Asia, a small number of Africans migrated along the Arabian Peninsula and Indian coast in South Asia and finally arrived in Australia [46]. The inverted allele number of Americans, which was between that of Africans and Europeans, is possibly due to both the African and European ancestry of the American populations. It is known that long-term migration can cause genomic variations [47]. Thus, we inferred that after the Out of Africa migration of modern human ancestors and hybridization among the four non-African super-populations, the Africans introduced some inverted alleles into the ancestors of the other four super-populations. It is speculated that the differential co-evolution of MI lineages with different but closely related ancestral populations and subsequent evolution of MIs in parallel with the introgression of archaic alleles into the genomes of modern human ancestors may have been largely responsible for the present-day variant counts of MIs in multiple populations. Therefore, our results may provide evidence for the Out of Africa hypothesis.

\subsection{Phylogenetic Tree Analysis and PCA}

In the phylogenetic analysis of the 26 populations, geographical distributions and migrations affected the MIs. African and American populations were clustered on a branch in the phylogenetic tree, perhaps because African Americans are the largest racial minority [48] and mixed heritage is common in America. Specifically, the populations on the East Coast of America and in Western Africa were clustered on another branch. This cluster may be due to the black slave trade from Western Africa to the East Coast of America [49]. The populations on the West Coast of America and in Eastern Africa were more closely related than the other populations. This closeness is consistent with the migration of people from Eastern Africa to the West Coast of America [50]. We found that European and South Asian populations were clustered on one branch (as shown in Fig. 4), which has 
also been reported in other studies focusing on CNVs [51] and retro-duplications [52]. The specificity of the Finnish population (FIN) can be interpreted in terms of multiple genetic components and demographic factors, such as isolation and migration, as reflected in their distinctive distribution among the European populations in another study [53]. Moreover, our finding that Puerto Ricans from Puerto Rico (PUR) are closely related to populations in East Asia is reasonable because some Peruvians are Asian immigrants [54]. These MIs used in phylogenetic tree analysis and PCA could also be used to cluster different super-populations, showing that these MIs represented the different genomic backgrounds of the studied populations. The results of the phylogenetic analysis of the 26 populations are consistent with the corresponding migration history and composition of these populations.

\subsection{Comparative Analysis with Non-Human Primates}

Evidence for the Out of Africa hypothesis also stems from a comparison between the MIs of non-human primates and those of humans. Africans shared more inverted alleles with the 7 non-human primates than did the other 4 superpopulations, which shared only a few. The highest ratio of inverted alleles overlap observed between the non-human primates and Africans might be because Africans inherited these shared ancestry alleles from the common ancestor of non-human primates and humans in ancient times. It has been reported that genetic interchange among human populations, in terms of both periodic gene flow restricted by geographical space and events of main population expansion, contributed to interbreeding but not replacement [55]. Thus, it may be assumed that after the Out of Africa geographic movement of modern humans, the African ancestral population spread the distribution of ancestry alleles that are shared with the 7 non-human primates, and some of these ancestry alleles now survive in contemporary populations. Indeed, periodic genetic interchange occurred among human super-populations, in terms of both frequent gene flow constrained by geographical distance and population expansion events causing interbreeding. Because not all of the ancestry alleles in Africans would have been introduced into the other four super-populations, a smaller number of ancestry alleles remained in the other four super-populations, while some individuals finally inherited the derived allele. However, further sophisticated fossil and genetic data are needed to confirm this inference. MIs overlapped with 3 genes, MYH14, GRM7, and DFNA5, all of which are related to hearing [56-58], in both humans and non-human primates. This finding suggests that the inverted alleles in these 3 genes may have existed in the common ancestors of humans and non-human primates and were subsequently conserved in some non-human primates and some human super-populations. Therefore, the super-populations that inherited the inverted alleles may differ from others in terms of hearing.

Among the common 76 inverted alleles in the five superpopulations and 34 overlapped with genes. The inverted alleles shared by all the five super-populations could be a result of incompleteness of the current human reference assembly, while some of these regions could have been previously reported as SNPs because of the limited understanding of MIs. In addition, the human reference assembly has been reported to contain rare alleles, according to the Genome Reference Consortium. Accordingly, MIs are potential candidates in future updates of the human reference assembly.

\subsection{Effects of Mls on Human Health}

Here, we focused more on the effects of MIs on human evolution. However, as we analyzed the genes that overlapped with MIRs, we found that MIs may be associated with human health. Though the functions of the intronic regions and UTRs affected by the MIs detected with limited samples in this study are not yet clear, these MIs may benefit studies of the relation between variation and health in the future. In addition, the inverted alleles found in only 1 super-population, especially those overlapping with gene regions, may increase susceptibility to some diseases. Because of limited samples from normal individuals with no important genetic diseases in the $1 \mathrm{KGP}$, the MIs in this study are candidates for future studies. Accordingly, more MIs from samples with diseases are needed to explore the correlation between MIs and human health. Nevertheless, we expect that personalized medical information will help us better understand the relationship between MIs and health, as well as unveil disease mechanisms, in the future.

\section{Conclusion}

To the best of our knowledge, this is the first study to analyze MIs in humans at the population scale. Our analysis of MIs with 1KGP data improves our understanding of human genetic diversity and evolution. The comparative analysis of MIs at the population, super-population, and species scales is, thus, expected to contribute to further implementation of human evolutionary theory. The future use of large-scale sophisticated fossil data and archeological materials to analyze the ages of MI polymorphisms will be informative in understanding the behavior of MIs as genetic markers and for better reconstructing human evolutionary history. 


\section{Materials and Methods}

\subsection{Dataset}

To elucidate the features of MIs among human genomes, we collected BAM files of the unmapped reads of 2427 samples from the most recent release of the 1KGP [27]. Among all the samples, 490 did not contain MIs and were thus excluded. The 1937 included samples of unmapped short reads with a total file size of $742 \mathrm{~GB}$, which covered all 26 populations, were categorized into five super-populations: East Asians [Chinese Dai in Xishuangbanna, China (CDX); Han Chinese in Beijing, China (CHB); Southern Han Chinese (CHS); Japanese in Tokyo, Japan (JPT); and Kinh in Ho Chi Minh City, Vietnam (KHV)], South Asians [Bengali from Bangladesh (BEB); Gujarati Indians from Houston, Texas (GIH); Indian Telugu from the UK (ITU); Punjabi from Lahore, Pakistan (PJL); and Sri Lankan Tamil from the UK (STU)], Europeans [Utah Residents (CEPH) with Northern and Western Ancestry (CEU), Finnish in Finland (FIN), British in England and Scotland (GBR), Iberian Population in Spain (IBS), and Toscani in Italia (TSI)], Americans [Colombians from Medellin, Colombia (CLM); Mexican Ancestry from Los Angeles, USA (MXL); Peruvians from Lima, Peru (PEL); and Puerto Ricans from Puerto Rico (PUR)], and Africans [African Caribbeans in Barbados (ACB); Americans of African Ancestry in SW USA (ASW); Esan in Nigeria (ESN); Gambians in Western Divisions in the Gambia (GWD); Luhya in Webuye, Kenya (LWK); Mende in Sierra Leone (MSL); and Yoruba in Ibadan, Nigeria (YRI)]. The full list of all 1937 sample names classified into 26 populations is shown in Table S1.

For further comparative analysis between humans and other primates, we also downloaded the hg 19 human reference genome and the pairwise alignment data of 7 nonhuman primate assemblies from the UCSC Genome Browser (https://hgdownload.soe.ucsc.edu/downloads.html) [34]. We used data version panTro4 for the human/chimpanzee alignment, gorGor3 for the human/gorilla alignment, ponAbe 2 for the human/orangutan alignment, nomLeu1 for the human/ gibbon alignment, papHam1 for the human/baboon alignment, rheMac3 for the human/rhesus macaque alignment, and saiBol1 for the human/squirrel monkey alignment, all of which were downloaded from the UCSC Genome Browser (https://genome.ucsc.edu/).

\subsection{Detection and Annotation}

To detect MIs in data from the $1 \mathrm{KGP}$, we applied the software MID [13] with default parameters and mapped all unmapped sequencing reads to the human reference genome (hg19) [34]. We chose MID because our previous investigation showed that MID was capable of efficiently identifying MIs from unmapped short reads through inversion reading and reference genome mapping. Specifically, the same MI occurring in multiple short reads of the same individual was counted as 1 MI during MID detection. After detecting MIs, we annotated them with both gene and correlated translated protein functions via the GENCODE database (https://www.gencodegenes.org/) [59], GeneCards database (https://www.genecards.org/) [60], and Metascape software [61]. The candidate promoter regions were defined as the regions within $2000 \mathrm{bp}$ upstream (for plus strand genes) or downstream (for minus strand genes) of gene regions. For the MIs overlapping with the CDS regions listed in Table S5, whenever possible, we used the annotations for SVs and SNPs from the UCSC Genome Browser (https://genome.ucsc.edu/). The SVs and SNPs were annotated by the database of 1000G Ph3 Vars [27], dbSNP [31], ExAC [32], and ClinVar [33]. We detected the MIs of nonhuman primates in the aligned data using the searchUMI tool [44] because it is able to identify inversions ranging from 5 to $125 \mathrm{bp}$ in length, which is the approximate length of MIs. During detection, the parameter pd was set at 0.0137 for panTro4, 0.0175 for gorGor3, 0.034 for ponAbe2, 0.029 for nomLeu 1, 0.066 for papHam 1, 0.065 for rheMac3, and 0.123 for saiBol1. Furthermore, based on the definition of MIs in this study, we removed inversions that were shorter than $10 \mathrm{bp}$.

\subsection{Statistical analyses of MI diversity}

Because sample number varied by population, diversity would be better reflected by the average number of inverted alleles per individual among super-populations or populations than by the total inverted allele number of each superpopulation or population. To address this point, we defined parameter $\overline{C_{p}}$ to represent the average count of inverted alleles per individual among super-populations or populations as follows:

$\overline{C_{p}}=\frac{\sum_{i=1}^{\mathrm{N}_{p}} \mathrm{C}_{i}}{\mathrm{~N}_{\mathrm{p}}}$,

where $\mathrm{C}_{i}$ is the count of inverted alleles per individual, and $\mathrm{Np}$ is the total number of individuals in a population or super-population. The MI counts in each individual among the five super-populations, which were used to calculate the mean and SE of inverted allele numbers per individual in the five super-populations, are listed in Table S11.

We used MIs but not MIRs to calculate the average inverted allele number because the MIR has merged a few MIs as one, and thus, MIR numbers cannot truly reflect the specific level of inverted allele numbers for each population. 
For the same reason, the phylogenetic tree and PCA analysis were built and performed based on the matrix of MIs. However, only when analyzing the inverted alleles shared by the five super-populations with the Venn diagram did we adopt MIRs for analysis, because in this analysis, we expected to see the overlapped alleles in the five super-populations and then to ascertain whether these overlapped regions had some characteristics.

\subsection{Population structure based on Mls}

To analyze the population structure based on MIs among all populations from an evolutionary and comparative viewpoint, we next calculated a matrix with rows denoting MIRs and columns denoting all 26 human populations. Each element in this matrix represented the sum of inverted alleles included in the corresponding MIR within a given population. The matrices for constructing the phylogenetic tree based on all MIs are shown in Table S6. In addition, the matrices for constructing the phylogenetic tree based only on MIs in gene regions are provided in Table S7. With these matrices, we constructed a phylogenetic tree with the neighbor-joining algorithm via the $\mathrm{R}$ language. MIR sharing was analyzed by counting the number of shared MIRs among the five super-populations. The distances used in the neighborjoining algorithm were Euclidean distances between every pair of column vectors, i.e., the distance between two populations was defined as the average MIR distance between pairs of individuals from the two populations. In the same way, the phylogenetic tree of the five super-populations was also constructed based on the neighbor-joining clustering method with the MIs obtained in gene regions (Table S7).

MIR sharing was analyzed by counting the number of shared MIRs among the five super-populations. The population structure of the 26 populations was also visualized through PCA with the R programming language to determine whether the distribution of MIs was based on geographical location and migration history. For the PCA, we applied the same matrix (Table S6) used in the phylogenetic tree analysis. In addition, we regarded the 2140 MIRs as the principal components in the PCA.

Acknowledgements The authors thank Dr. Jian Ma of Carnegie Mellon University, and Dr. Qi Wang, Dr. Xiaoqi Wang, Dr. Yongchu Liu, Dr. Jiangtao Guo, Dr. Binbin Lai, and Dr. Yang Li for their beneficial discussions and assistance with this paper.

Author contributions HQZ conceived and designed the experiments. LQ, LTW and FFH performed MIs detection and computational analyses. LQ, FFH and LSY wrote computer code for the sequence analysis. YLH also contributed partly to computational analyses. HQZ, LQ, LTW and LSY wrote and revised the manuscript. All authors participated in data analyses. All authors reviewed the manuscript.
Funding This work was supported by the National Key Research and Development Program of China (2017YFC1200205), the National Natural Science Foundation of China (31671366 and 91231119), and the Special Research Project of 'Clinical Medicine $+X$ ' by Peking University. Part of the analysis was performed on the High Performance Computing Platform of the Center for Life Science of Peking University.

Data Availability The data and results are presented within the manuscript and Supplementary files.

Code Availability The codes used in this manuscript were conducted in $\mathrm{R}$ packages

\section{Compliance with Ethical Standards}

Conflict of Interest The authors declare no competing interests.

Open Access This article is licensed under a Creative Commons Attribution 4.0 International License, which permits use, sharing, adaptation, distribution and reproduction in any medium or format, as long as you give appropriate credit to the original author(s) and the source, provide a link to the Creative Commons licence, and indicate if changes were made. The images or other third party material in this article are included in the article's Creative Commons licence, unless indicated otherwise in a credit line to the material. If material is not included in the article's Creative Commons licence and your intended use is not permitted by statutory regulation or exceeds the permitted use, you will need to obtain permission directly from the copyright holder. To view a copy of this licence, visit http://creativecommons.org/licenses/by/4.0/.

\section{References}

1. Puig M, Casillas S, Villatoro S, Cáceres M (2015) Human inversions and their functional consequences. Brief Funct Genom 14:369-379. https://doi.org/10.1093/bfgp/elv020

2. Yunis JJ, Dunham K (1980) The striking resemblance of highresolution G-banded chromosomes of man and chimpanzee. Science 208:1145-1148. https://doi.org/10.1126/science.7375922

3. Salm MP et al (2012) The origin, global distribution, and functional impact of the human 8p23 inversion polymorphism. Genome Res 22:1144-1153. https://doi.org/10.1101/gr.12603 7.111

4. Stefansson $\mathrm{H}$ et al (2005) A common inversion under selection in Europeans. Nat Genet 37:129-137. https://doi.org/10.1038/ng150 8

5. Kidd JM et al (2008) Mapping and sequencing of structural variation from eight human genomes. Nature 453:56-64. https://doi. org/10.1038/nature06862

6. Korbel JO et al (2007) Paired-end mapping reveals extensive structural variation in the human genome. Science 318:420-426. https://doi.org/10.1126/science.1149504

7. Baker M et al (1999) Association of an extended haplotype in the tau gene with progressive supranuclear palsy. Hum Mol Genet 8:711-715. https://doi.org/10.1093/hmg/8.4.711

8. Oliveira SA et al (2004) Linkage disequilibrium and haplotype tagging polymorphisms in the Tau H1 haplotype. Neurogenetics 5:147-155. https://doi.org/10.1007/s10048-004-0180-5

9. Jobling MA et al (1998) A selective difference between human Y-chromosomal DNA haplotypes. Curr Biol 8:1391-1394. https ://doi.org/10.1016/S0960-9822(98)00020-7

10. Kurotaki N et al (2002) Haploinsufficiency of NSD1 causes Sotos syndrome. Nat Genet 30:365-366. https://doi.org/10.1038/ng863 
11. Kolb J et al (2009) Cruciform-forming inverted repeats appear to have mediated many of the microinversions that distinguish the human and chimpanzee genomes. Chromosom Res 17:469-483. https://doi.org/10.1007/s10577-009-9039-9

12. Hou M, Yao P, Antonou A, Johns MA (2011) Pico-inplace-inversions between human and chimpanzee. Bioinformatics 27:32663275. https://doi.org/10.1093/bioinformatics/btr566

13. He F, Li Y, Tang YH, Ma J, Zhu H (2016) Identifying microinversions using high-throughput sequencing reads. BMC Genomics 17:4. https://doi.org/10.1186/s12864-015-2305-7

14. Chaisson MJ, Raphael BJ, Pevzner PA (2006) Microinversions in mammalian evolution. Proc Natl Acad Sci USA 103:1982419829. https://doi.org/10.1073/pnas.0603984103

15. Hoffmann AA, Rieseberg LH (2008) Revisiting the impact of inversions in evolution: from population genetic markers to drivers of adaptive shifts and speciation? Annu Rev Ecol Evol S 39:2142. https://doi.org/10.1146/annurev.ecolsys.39.110707.173532

16. Fischer G, Rocha EP, Brunet F, Vergassola M, Dujon B (2006) Highly variable rates of genome rearrangements between hemiascomycetous yeast lineages. PLoS Genet 2:e32. https://doi. org/10.1371/journal.pgen.0020032

17. Jones FC et al (2012) The genomic basis of adaptive evolution in three spine sticklebacks. Nature 484:55-61. https://doi. org/10.1038/nature10944

18. Kennington WJ, Partridge L, Hoffmann AA (2006) Patterns of diversity and linkage disequilibrium within the cosmopolitan inversion in (3R) Payne in Drosophila melanogaster are indicative of coadaptation. Genetics 172:1655-1663. https://doi. org/10.1534/genetics.105.053173

19. Bourque G, Zdobnov EM, Bork P, Pevzner PA, Tesler G (2005) Comparative architectures of mammalian and chicken genomes reveal highly variable rates of genomic rearrangements across different lineages. Genome Res 15:98-110. https://doi.org/10.1101/ gr.3002305

20. Braun EL et al (2011) Homoplastic microinversions and the avian tree of life. BMC Evol Biol 11:141. https://doi. org/10.1186/1471-2148-11-141

21. Lyon MF (2003) Transmission ratio distortion in mice. Annu Rev Genet 37:393-408. https://doi.org/10.1146/annurev.genet .37.110801.143030

22. Alves MJ, Lopes MA, Chikhi L, Amorim A (2012) On the structural plasticity of the human genome: chromosomal inversions revisited. Curr Genom 13:623-632. https://doi.org/10.2174/13892 0212803759703

23. Sanders AD, Hills M, Porubský D, Guryev V, Falconer E, Lansdorp PM (2016) Characterizing polymorphic inversions in human genomes by single-cell sequencing. Genome Res 26(11):15751587. https://doi.org/10.1101/gr.201160.115

24. Audano PA et al (2019) Characterizing the major structural variant alleles of the human genome. Cell 176(3):663-675. https://doi. org/10.1016/j.cell.2018.12.019

25. Levy-Sakin M et al (2019) Genome maps across 26 human populations reveal population-specific patterns of structural variation. Nat Commun 10:1025. https://doi.org/10.1038/s41467-019-08992 $-7$

26. Giner-Delgado C et al (2019) Evolutionary and functional impact of common polymorphic inversions in the human genome. Nat Commun 10:4222. https://doi.org/10.1038/s41467-019-12173-x

27. Auton A et al (2015) A global reference for human genetic variation. Nature 526(7571):68-74. https://doi.org/10.1038/nature1539 3

28. Sudmant PH et al (2015) An integrated map of structural variation in 2,504 human genomes. Nature 526:75-81. https://doi. org/10.1038/nature 15394
29. Stringer CB, Andrews P (1988) Genetic and fossil evidence for the origin of modern humans. Science 239:1263-1268. https:// doi.org/10.1126/science. 3125610

30. Yang TL et al (2008) Genome-wide copy-number-variation study identified a susceptibility gene, UGT2B17, for osteoporosis. Am J Hum Genet 83:663-674. https://doi.org/10.1016/j. ajhg.2008.10.006

31. Sherry ST et al (2001) dbSNP: the NCBI database of genetic variation. Nucleic Acids Res 29:308-311. https://doi.org/10.1093/ nar/29.1.308

32. Karczewski KJ et al (2017) The ExAC browser: displaying reference data information from over 60000 exomes. Nucleic Acids Res 45:D840-D845. https://doi.org/10.1093/nar/gkw971

33. Landrum MJ et al (2016) ClinVar: public archive of interpretations of clinically relevant variants. Nucleic Acids Res 44:D862-D868. https://doi.org/10.1093/nar/gkv1222

34. Haeussler M et al (2019) The UCSC genome browser database: 2019 update. Nucleic Acids Res 47(D1):D853-D858. https://doi. org/10.1093/nar/gky1095

35. Rotimi CN, Bentley AR, Doumatey AP, Chen G, Shriner D, Adeyemo A (2017) The genomic landscape of African populations in health and disease. Hum Mol Genet 26:R225-R236. https://doi. org $/ 10.1093 / \mathrm{hmg} / \mathrm{ddx} 253$

36. Lek $\mathrm{M}$ et al (2016) Analysis of protein-coding genetic variation in 60,706 humans. Nature 536:285. https://doi.org/10.1038/natur e19057

37. Jo BS, Choi SS (2015) Introns: the functional benefits of introns in genomes. Genom Inform 13(4):112. https://doi.org/10.5808/ GI.2015.13.4.112

38. Hughes TA (2006) Regulation of gene expression by alternative untranslated regions. Trends Genet 22(3):119-122. https://doi. org/10.1016/j.tig.2006.01.001

39. $\mathrm{Xu} \mathrm{K}$ et al (2015) Genome wide association study for maximum number of alcoholic drinks in European Americans and African Americans. Alcohol Clin Exp Res 39:1137-1147. https://doi. org/10.1111/acer.12751

40. ENCODE Project Consortium (2004) The ENCODE (ENCyclopedia of DNA elements) project. Science 306(5696):636-640. https ://doi.org/10.1126/science.1105136

41. Pundhir S, Hannibal TD, Bang-Berthelsen $\mathrm{CH}$, Wegener AMK, Pociot F, Holmberg D, Gorodkin J (2014) Spatially conserved regulatory elements identified within human and mouse Cd247 gene using high-throughput sequencing data from the ENCODE project. Gene 545(1):80-87. https://doi.org/10.1016/j. gene.2014.05.004

42. Quinn EM, Hill M, Anney R, Gill M, Corvin AP, Morris DW (2010) Evidence for cis-acting regulation of ANK3 and CACNA1C gene expression. Bipolar Disord 12(4):440-445. https:// doi.org/10.1111/j.1399-5618.2010.00817.x

43. Yuan A et al (2012) ANK3 as a risk gene for schizophrenia: new data in Han Chinese and meta analysis. Am J Med Genet B Neuropsychiatr Genet 159B(8):997-1005. https://doi.org/10.1002/ ajmg.b.32112

44. Hara Y, Imanishi T (2011) Abundance of ultramicro inversions within local alignments between human and chimpanzee genomes. BMC Evol Biol 11:308. https://doi. org/10.1186/1471-2148-11-308

45. Nei M, Livshits G (1989) Genetic relationships of Europeans, Asians and Africans and the origin of modern Homo sapiens. Hum Hered 39:276-281. https://doi.org/10.1159/000153872

46. Macaulay V et al (2005) Single, rapid coastal settlement of Asia revealed by analysis of complete mitochondrial genomes. Science 308(5724):1034-1036. https://doi.org/10.1126/science.1109792

47. Hollfelder N, Schlebusch CM, Günther T, Babiker H, Hassan HY, Jakobsson M (2017) Northeast African genomic variation shaped by the continuity of indigenous groups and Eurasian 
migrations. PLoS Genet 13:e1006976. https://doi.org/10.1371/ journal.pgen.1006976

48. Snipp CM (1996) Understanding race and ethnicity in rural America. Rural Sociol 61(1):125-142. https://doi. org/10.1111/j.1549-0831.1996.tb00613.x

49. Fage JD (1969) Slavery and the slave trade in the context of West African history. J Afr History 10:393-404. https://doi. org/10.1017/S0021853700036343

50. Djamba YK (1999) African immigrants in the United States: a socio-demographic profile in comparison to native blacks. J Asian Afr Stud 34(2):210-215. https://doi.org/10.1177/0021909699 03400204

51. Lou $\mathrm{H}$ et al (2011) A map of copy number variations in Chinese populations. PLoS ONE 6:e27341. https://doi.org/10.1371/journ al.pone.0027341

52. Zhang Y, Li S, Abyzov A, Gerstein MB (2017) Landscape and variation of novel retroduplications in 26 human populations. PLoS Comput Biol 13:e1005567. https://doi.org/10.1371/journ al.pcbi.1005567

53. Palo JU, Ulmanen I, Lukka M, Ellonen P, Sajantila A (2009) Genetic markers and population history: Finland revisited. Eur J Hum Genet 17:1336-1346. https://doi.org/10.1038/ejhg.2009.53

54. Takenaka A (2004) The Japanese in Peru: history of immigration, settlement, and racialization. Lat Am Perspect 31(3):77-98. https ://doi.org/10.1177/0094582X04264745
55. Templeton A (2002) Out of Africa again and again. Nature 416:45. https://doi.org/10.1038/416045a

56. Kim BJ, Kim AR, Han JH, Lee C, Oh DY, Choi BY (2017) Discovery of MYH14 as an important and unique deafness gene causing prelingually severe autosomal dominant nonsyndromic hearing loss. J Gene Med 19(4):e2950. https://doi.org/10.1002/ jgm. 2950

57. Friedman RA et al (2009) GRM7 variants confer susceptibility to age-related hearing impairment. Hum Mol Genet 18:785-796. https://doi.org/10.1093/hmg/ddn402

58. De Beeck KO et al (2011) The DFNA5 gene, responsible for hearing loss and involved in cancer, encodes a novel apoptosisinducing protein. Eur J Hum Genet 19:965-973. https://doi. org/10.1038/ejhg.2011.63

59. Harrow $\mathbf{J}$ et al (2012) GENCODE: the reference human genome annotation for the ENCODE project. Genome Res 22:1760-1774. https://doi.org/10.1101/gr.135350.111

60. Safran M et al (2010) GeneCards Version 3: the human gene integrator. Database. https://doi.org/10.1093/database/baq020

61. Tripathi $\mathrm{S}$ et al (2015) Meta-and orthogonal integration of influenza "OMICs" data defines a role for UBR4 in virus budding. Cell Host Microbe 18:723-735. https://doi.org/10.1016/j. chom.2015.11.002 